\title{
Novel tumor-suppressor FOXN3 is downregulated in adult acute myeloid leukemia
}

\author{
HANG HE，JINJING ZHANG，YI QU，YUE WANG，YAN ZHANG，XIAOJING YAN，YAN LI and RUI ZHANG \\ Department of Hematology, The First Affiliated Hospital of China Medical University, \\ Shenyang, Liaoning 110001, P.R. China
}

Received August 8, 2018; Accepted February 28, 2019

DOI: $10.3892 / \mathrm{ol} .2019 .10424$

\begin{abstract}
Forkheadbox protein N3 (FOXN3) is a transcriptional repressor involved in cell cycle regulation and tumorigenesis. Abnormalities in gene structure and epigenetics of FOXN3 are closely associated with the occurrence of hematological malignancies; however, its involvement in the pathogenesis of acute myeloid leukemia (AML) remains unknown. The present study aimed to examine the potential significance of FOXN3 in AML. FOXN3 expression levels were examined in patients with AML and AML cell lines, and its clinical significance in AML was evaluated. FOXN3-overexpressing AML cell lines were established, and the biological function of FOXN3 was detected by flow cytometry and a Cell Counting Kit- 8 assay. A significant decrease in FOXN3 expression levels was observed in patients with AML and in the AML cell lines in vitro. FOXN3 expression levels were associated with the number of leukocytes in patients. FOXN3 overexpression may inhibit cell proliferation in AML cell lines, induce cell cycle S-phase arrest and promote apoptosis in OCI-AML3 and THP-AML cells. The present study provided insight into how FOXN3 may serve as a novel tumor suppressor in AML.
\end{abstract}

\section{Introduction}

Acute myeloid leukemia (AML) originates from hematopoietic stem/progenitor cells characterized by a high degree of heterogeneity. The diagnostic criteria for AML has shifted from the French-American-British (FAB) criteria for morphological diagnosis to the World Health Organization (WHO) criteria, which includes alterations in cytogenetic and molecular biological characterization $(1,2)$, demonstrating the importance of cytogenetic and molecular biological characteristics as important prognostic factors of AML. Therefore, identifica-

Correspondence to: Dr Rui Zhang, Department of Hematology, The First Affiliated Hospital of China Medical University, 155 Nanjing North Street, Shenyang, Liaoning 110001, P.R. China E-mail: hemozerro2008@hotmail.com

Key words: acute myeloid leukemia, cell cycle, forkhead box protein N3, prognosis, survival analysis tion of specific genes involved in leukemogenesis may improve the diagnosis and treatment of AML.

In a previous study, the genomes of 24 patients with acute monocytic leukemia were scanned by high-resolution array comparative genome hybridization (aCGH) technology. A total of two patients presented with a deletion of chromosome 14 involving the forkhead box protein $\mathrm{N}$ (FOXN)3 gene (3). FOXN3 was first identified by Pati et al (4) in a yeast cell with multiple checkpoint mutations. It is a subtype of the forkhead box protein (FOX) transcription factor family, and is referred to as checkpoint suppressor $1(5,6)$. FOXN3 possesses an important role in the development of cells and tissues $(7,8)$. It is crucial for the development of brain cartilage and indirectly influences the development of muscle morphology (7). During cellular DNA damage, FOXN3 may prolong cell survival by inducing cell quiescence $(9,10)$. As a DNA damage response protein, FOXN3 restored cell cycle arrest (S-phase) in the mutant fruit fly (11). Previous studies demonstrated the ability of FOXN3 to decrease the malignancy of tumors, including in liver cancer, lung cancer, colon cancer and certain hematological malignancies (12-15). However, the role of FOXN3 in AML is not yet understood, to the best of the authors' knowledge. It is hypothesized that FOXN3 is abnormally expressed in patients with AML and may serve as a tumor suppressor gene contributing to the transformation of leukemia.

In the present study, FOXN3 expression and its association with clinicopathological features of AML were investigated in patients with AML. The role of FOXN3 in promoting an AML phenotype was further studied in AML cell lines in vitro.

\section{Materials and methods}

Patient samples and cell lines. A total of 53 patients with recently diagnosed AML (47 patients were confirmed with AML at diagnosis and 6 patients were confirmed at relapse), who were admitted to The First Affiliated Hospital of China Medical University (Shenyang, China) between March 2012 and May 2013 and fulfilled the diagnostic criteria of FAB and WHO $(1,2)$, were included in the present study. Patients who were $\leq 14$ years old or patients with secondary AML (transformed from myelodysplastic syndrome or myeloproliferative disease) were excluded from the present study. The clinical characteristics of patients are summarized in Table I. The treatment response was evaluated as previously described (3). 
The definition of overall survival (OS) refers to the time between randomization and mortality. Relapse-free survival (RFS) refers to the time between complete remission (CR) and disease recurrence. The present study was approved by The Ethics Committee of The First Affiliated Hospital of China Medical University. Prior to data collection, written informed consent for participation in the present study and for publishing the present results was obtained from each participant. Bone marrow samples were collected from 14 normal donors between May and August 2017 to serve as healthy controls. The average age was 45 years old with 8 male and 6 female participants.

Human normal 293T cells and AML cell lines OCI-AML3 (OA3), NB4, U937, JURKAT and K562 were purchased from The American Type Culture Collection (Manassas, VA, USA), and KASUMI-1 and THP-AML (THP-1) cell lines were purchased from The Type Culture Collection of the Chinese Academy of Medical Sciences (Shanghai, China). Cells were cultured with RPMI-1640 medium (Thermo Fisher Scientific, Inc., Waltham, MA, USA) containing 10\% fetal bovine serum (Gibco; Thermo Fisher Scientific, Inc.) at $37^{\circ} \mathrm{C}$ in a humidified incubator with $5 \% \mathrm{CO}_{2}$.

Reverse transcription-quantitative polymerase chain reaction (RT-qPCR). Total RNA was extracted from bone marrow specimens of patients with AML and AML cell lines using TRIzol $^{\circledR}$ (Invitrogen; Thermo Fisher Scientific, Inc.). RT was performed at $37^{\circ} \mathrm{C}$ for $15 \mathrm{~min}$ and then $85^{\circ} \mathrm{C}$ for $15 \mathrm{~min}$ using a genomic DNA Eraser RT kit (Takara Bio, Inc., Otsu, Japan). The relative FOXN3 expression levels were calculated and normalized using the comparative quantification cycle $2^{-\Delta \Delta C q}$ method relative to an endogenous control GAPDH according to a previous study (16). RT-qPCR was performed as previously described by Zhang et al (3). The primer sequences are listed in Table II.

Immunohistochemistry. To assess the FOXN3 protein expression levels in leukemia blasts, immunohistochemical staining was performed using the immunoperoxidase technique and an avidin-biotin-peroxidase complex as previously described (17). Cytospin smears of bone marrow cells from AMLs with a blast ratio $>80 \%$ and normal controls (bone marrow transplant donor) were fixed in formalin acetone (3\%) at room temperature for $3 \mathrm{~min}$. The specimens were incubated with peroxidase (reagent 1) from a streptavidin-peroxidase (SP) link detection kit [Biotin-Streptavidin horseradish peroxidase (HRP) Detection Systems; cat. no. SP9001; OriGene Technologies, Inc., Rockville, MD, USA) blocking enzyme for $20 \mathrm{~min}$ and then incubated with normal goat serum (reagent 2 from the kit) for blocking at room temperature for $15 \mathrm{~min}$, followed by incubation with rabbit anti-human antibodies against FOXN3 protein (cat. no. HPA059209; 1:50 in PBS; Sigma-Aldrich; Merck KGaA, Darmstadt, Germany) at $37^{\circ} \mathrm{C}$ for $60 \mathrm{~min}$. Biotinylated goat anti-rabbit immunoglobulin $\mathrm{G}$ (reagent 3 from the kit) was used as the secondary antibody, incubated at room temperature for $15 \mathrm{~min}$, and detected using the SP complex (reagent 4 from the kit). The specimens were counterstained with hematoxylin for $20 \mathrm{sec}$ at room temperature. Antibody reactivity was detected with vector brown avidin-biotin-peroxidase
Table I. Clinicopathological characteristics of patients with acute myeloid leukemia.

\begin{tabular}{|c|c|c|}
\hline $\begin{array}{l}\text { Clinicopathological } \\
\text { characteristics }\end{array}$ & $\begin{array}{l}\text { Number } \\
\text { of patients }\end{array}$ & $\%$ \\
\hline \multicolumn{3}{|l|}{ Sex } \\
\hline Male & 27 & 50.94 \\
\hline Female & 26 & 49.06 \\
\hline \multicolumn{3}{|l|}{ Age, years } \\
\hline$\leq 60$ & 40 & 75.47 \\
\hline$>60$ & 13 & 24.53 \\
\hline \multicolumn{3}{|c|}{ White blood cell count, $10^{9} / 1$} \\
\hline$<30$ & 39 & 73.58 \\
\hline$\geq 30$ & 14 & 26.42 \\
\hline \multicolumn{3}{|l|}{ Hemoglobin, g/l } \\
\hline$\leq 80$ & 37 & 69.81 \\
\hline$>80$ & 16 & 30.19 \\
\hline \multicolumn{3}{|l|}{ Platelet, $10^{9} / 1$} \\
\hline$\leq 50$ & 36 & 67.92 \\
\hline$>50$ & 17 & 32.08 \\
\hline \multicolumn{3}{|l|}{ Blasts, \% } \\
\hline$\leq 70$ & 27 & 50.94 \\
\hline$>70$ & 26 & 49.06 \\
\hline \multicolumn{3}{|l|}{ FAB subtype } \\
\hline M2 & 13 & 24.53 \\
\hline M3 & 8 & 15.09 \\
\hline M5 & 25 & 47.17 \\
\hline Other type & 7 & 13.21 \\
\hline \multicolumn{3}{|l|}{ Karyotype } \\
\hline Normal & 17 & 32.08 \\
\hline $\mathrm{t}(15 ; 17)$ & 8 & 15.09 \\
\hline $\mathrm{t}(8 ; 21)$ & 2 & 3.77 \\
\hline Complex & 11 & 20.76 \\
\hline Not available & 15 & 28.30 \\
\hline
\end{tabular}

FAB, French-American-British criteria for morphological diagnosis.

Table II. Primer sequences in reverse transcription-quantitative polymerase chain reaction.

\begin{tabular}{ll}
\hline Gene name & \multicolumn{1}{c}{ Sequence, 5'-3' $^{\prime}$} \\
\hline FOXN3 & F: TGCCAATCACTCCCATTGGG \\
& R: CCGCATCCGGCAGCTGG \\
GAPDH & F: CGGATTTGGTCGTATTGGG \\
& R:CTGGAAGATGGTGATGGGATT
\end{tabular}

FOXN3, forkhead box protein N3; F, forward; R, reverse.

substrate using an Olympus BX51 optical microscope (magnification, x1000). 
A
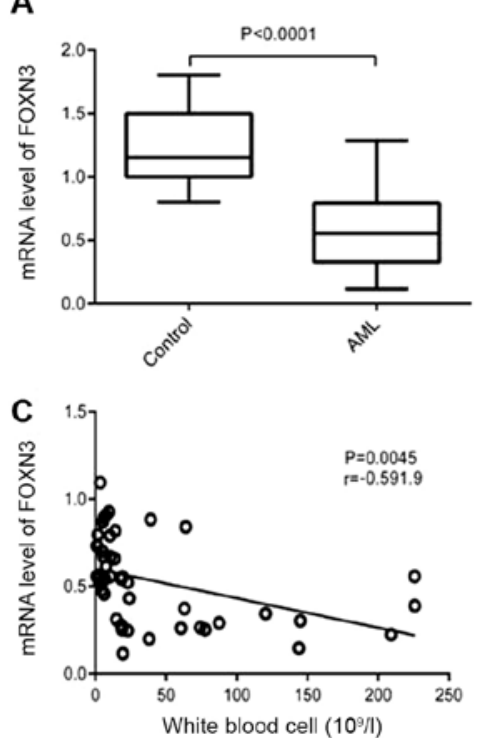

B
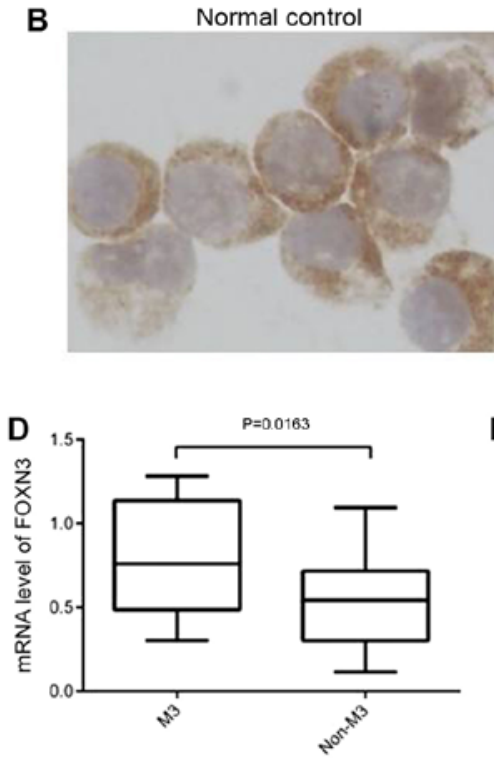

Patient with AML
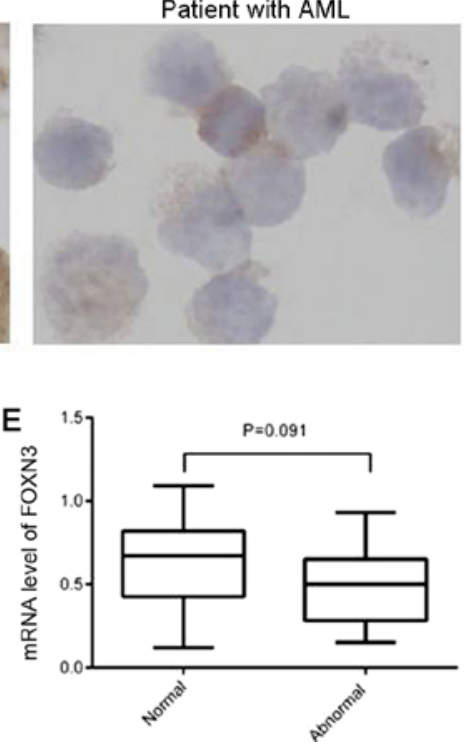

Figure 1. FOXN3 expression levels are decreased in AML samples. (A) mRNA expression levels of FOXN3 were significantly decreased in patients with AML compared with normal controls. $\mathrm{P}<0.0001$. (B) Representative images of immunohistochemistry of leukemia blasts to visualize FOXN3 expression levels in normal controls and patients with AML. Magnification, $x 40$. (C) Scatterplot representation of the correlation between FOXN3 expression levels and white blood cell count in AML samples. $\mathrm{r}=-0.5919$; $\mathrm{P}=0.0045$. (D) FOXN3 expression levels were significantly lower in patients classified with non-M3-subtype AML compared with patients classified with M3-subtype AML. P=0.0163. (E) FOXN3 expression levels in patients with normal and abnormal karyotypes. $\mathrm{P}=0.091$. FOXN3, forkhead box protein N3; AML, acute myeloid leukemia.

Construction of the lentiviral vector and cell transfection. The coding sequence of FOXN3 was amplified by RT-qPCR using oligo to design the primers (http://www.genepharma.com/) for the human FOXN3 gene. The primers were synthesized by Shanghai GenePharma Co., Ltd. (Shanghai, China) and were as follows: Forward 5'-TCTACTAGAGGATCCACTAGT GCCACCAGGGTCCAGTCATGCCTCCCAGTAAGAAG-3' and Reverse 3'-GTCGGGCCCTCTAGACYCGAGTTA ATtTtTtGtGgtTtCCTtTtGCTCTTt-5'. A PLEX vector (Shanghai GenePharma Co., Ltd.) was digested with SpeI and XhoI (Fermentas; Thermo Fisher Scientific, Inc.) and the FOXN3 gene was cloned into the vector. Positive clones were screened using ampicillin as a selection pressure (Sigma-Aldrich; Merck KGaA) and identified by Sanger sequencing on recombinant plasmid. For cells in suspension, a six-well plate was coated with fibronectin (ProSpec-Tany TechnoGene Ltd., East Brunswick, NJ, USA) and incubated at $37^{\circ} \mathrm{C}$ on a plate for at least $30 \mathrm{~min}$. A total of $5 \times 10^{5}$ cells/well were plated in a final volume of $1 \mathrm{ml}$ culture media. For infection, cells were plated onto 6 -well plates at $1 \times 10^{5}$ cells/well and infected with lentiviral stocks at a multiplicity of infection of 100 in the presence of polybrene $(8 \mu \mathrm{g} / \mathrm{ml}$; Sigma-Aldrich; Merck KGaA). Puromycin at a final concentration of $10 \mu \mathrm{g} / \mathrm{ml}$ (Sigma-Aldrich; Merck KGaA) was added to the media $72 \mathrm{~h}$ after transfection to select for stably transfected cells. The FOXN3 expression levels in stably transfected cell lines were examined by RT-qPCR and western blot analysis (18).

Western blot analysis. The cell lines were lysed using a whole protein extraction kit (Nanjing KeyGen Biotech, Co., Ltd., Nanjing, China). The total protein concentration was determined using a bicinchoninic acid protein kit, and $30 \mu \mathrm{g}$ protein was loaded per lane. The proteins were separated by $10 \%$ SDS-PAGE, transferred to a polyvinylidene fluoride membrane and blocked with $5 \%(\mathrm{~m} / \mathrm{v})$ fat-free milk for $60 \mathrm{~min}$ at room temperature. The membrane was incubated at $4^{\circ} \mathrm{C}$ overnight with the following primary antibodies: Anti-FOXN3 (1:2,500; cat. no. ab129453; Abcam, Cambridge, UK) and anti-GAPDH (1:1,000; cat. no. 5174; Cell Signaling Technology, Inc., Danvers, MA, USA). Specific bands were detected with an anti-rabbit immunoglobulin G, HRP-conjugated secondary antibody (1:2,000; cat. no. 7074; Cell Signaling Technology, Inc.) for $60 \mathrm{~min}$ at room temperature, and visualized using an Electro-Chemiluminescence plus chemiluminescence kit (cat. no. WBKLS0500; EMD Millipore, Billerica, MA, USA).

Cell growth analysis. The cells were counted, and the cell suspension was diluted to a final concentration of $2.5 \times 10^{4} / \mathrm{ml}$. The cell suspension was seeded into a $96-w e l l$ plate (100 $\mu \mathrm{l} /$ well). Subsequent to adding $10 \mu \mathrm{l}$ Cell Counting Kit-8 (CCK-8; Dojindo, Molecular Technologies, Inc., Kumamoto, Japan) solution to each well, the plates were incubated for $1 \mathrm{~h}$ in the incubator and the absorbance at $450 \mathrm{~nm}$ was measured with a microplate reader after 24,48 and $72 \mathrm{~h}$. Cell proliferation curves were plotted based on the absorbance values.

Flow cytometry. Apoptosis assays were performed using a flow cytometer with an Annexin V phycoerythrin/7-amino-actinomycin D cell apoptosis kit (cat. no. 550825; BD Biosciences, San Jose, CA, USA). The cell cycle was assessed using a cell cycle kit (cat. no. 559763; BD Biosciences) with propidium iodide. Specific steps for flow cytometry were as previously described (19). FlowJo software version 10 (TreeStar, Inc., Ashland, OR, USA) was used for flow cytometry analysis.

Statistical analysis. All statistical analyses were performed using SPSS 22.0 (IBM Corp., Armonk, NY, USA) and GraphPad Prism 6.0 software (GraphPad Software, Inc., 
Table III. Association between FOXN3 expression levels and the clinicopathological features of the patients.

\begin{tabular}{|c|c|c|}
\hline $\begin{array}{l}\text { Clinicopathological } \\
\text { characteristics }\end{array}$ & $\begin{array}{l}\text { FOXN3 } \\
\text { mRNA }^{a}\end{array}$ & P-value \\
\hline Sex & & 0.078 \\
\hline Male & $0.5070 \pm 0.2212$ & \\
\hline Female & $0.6431 \pm 0.2748$ & \\
\hline Age, years & & 0.582 \\
\hline Age $\leq 60$ & $0.5998 \pm 0.2850$ & \\
\hline Age $>60$ & $0.5520 \pm 0.2671$ & \\
\hline \multicolumn{3}{|l|}{ Peripheral blood count } \\
\hline White blood cell, $10^{9} / 1$ & & 0.005 \\
\hline$<30$ & $0.6859 \pm 0.1838$ & \\
\hline$\geq 30$ & $0.5151 \pm 0.2996$ & \\
\hline Hemoglobin, g/l & & 0.669 \\
\hline$\leq 80$ & $0.5970 \pm 0.2911$ & \\
\hline$>80$ & $0.5549 \pm 0.2607$ & \\
\hline Platelet, $10^{9} / 1$ & & 0.790 \\
\hline$\leq 50$ & $0.5689 \pm 0.2660$ & \\
\hline$>50$ & $0.5921 \pm 0.3003$ & \\
\hline Bone marrow, $\%$ & & 0.845 \\
\hline Blast $\leq 70$ & $0.5868 \pm 0.2773$ & \\
\hline Blast $>70$ & $0.5655 \pm 0.2772$ & \\
\hline FAB subtype & & 0.146 \\
\hline M2 & $0.5209 \pm 0.2529$ & \\
\hline M3 & $0.7884 \pm 0.3529$ & \\
\hline M5 & $0.5687 \pm 0.2560$ & \\
\hline Other & $0.4642 \pm 0.1934$ & \\
\hline Karyotype & & 0.122 \\
\hline Normal & $0.6178 \pm 0.2644$ & \\
\hline $\mathrm{t}(15 ; 17)$ & $0.7884 \pm 0.3528$ & \\
\hline $\mathrm{t}(8 ; 21)$ & $0.6073 \pm 0.0743$ & \\
\hline Complex & $0.5132 \pm 0.2390$ & \\
\hline Not available & $0.4584 \pm 0.2261$ & \\
\hline
\end{tabular}

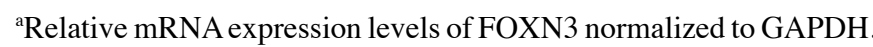
FOXN3, forkhead box protein N3; FAB, French-American-British criteria for morphological diagnosis.

La Jolla, CA, USA). The mRNA expression of FOXN3 was repeated three times and is presented as the mean \pm standard deviation. A Student's t-test was used to compare FOXN3 expression levels between patients with AML and healthy controls. Associations between FOXN3 expression levels and clinicopathological parameters were analyzed using non-parametric tests (Mann-Whitney $U$ test between two groups or Kruskal-Wallis test between three or more groups). A Spearman's rank correlation coefficient analysis was used to determine whether there was a correlation between FOXN3 expression levels and white blood cell (WBC) count. CR rates were compared between groups using a $\chi^{2}$ test. OS and the 2-year RFS curves were plotted using Kaplan-Meier curves, and the difference was tested using a log-rank test. Analysis of variance with a Tukey's post-hoc test was used to compare the mRNA expression difference between AML lines and 293T cell line, as well as the difference in cell proliferation, cell cycle and apoptosis between the control and FOXN3 overexpression cell lines. $\mathrm{P}<0.05$ was considered to indicate a statistically significant difference.

\section{Results}

FOXN3 expression levels are decreased in patients with AML. FOXN3 expression levels were detected in 53 bone marrow specimens of patients with AML present in the initial diagnosis and 14 normal healthy controls using RT-qPCR. FOXN3 expression levels were significantly decreased in patients with AML $(0.5764 \pm 0.03774)$ compared with the controls $(1.229 \pm 0.07865 ; \mathrm{P}<0.0001$; Fig. 1A). Decreased protein expression levels of FOXN3 were observed in six patients with decreased FOXN3 mRNA expression levels (representative example in Fig. 1B). The correlation between FOXN3 mRNA expression levels and the clinicopathological features of the 53 patients were assessed to further examine the significance of decreased FOXN3 expression levels (Table III). FOXN3 expression levels were significantly decreased in patients with WBC counts $>30 \times 10^{9} / 1$, and the expression level of FOXN3 was negatively correlated with the WBC count ( $r=-0.5919$; $\mathrm{P}=0.0045$; Fig. 1C). However, FOXN3 expression levels were not significantly decreased in patients with an increased blast ratio in bone marrow ( $\mathrm{P}=0.845$; Table III). Although no significant differences were observed in patients with different FAB subtypes ( $\mathrm{P}=0.146$; Table III), FOXN3 expression levels in patients with FAB non-M3-subtype $(0.5387 \pm 0.03645)$ were significantly decreased compared with patients with FAB M3-subtype (0.7884 $\pm 0.3529 ; \mathrm{P}=0.0163$; Fig. 1D). Furthermore, no statistically significant differences were identified in FOXN3 expression levels between normal and abnormal karyotypes in non-M3 patients $(\mathrm{P}=0.091$; Fig. 1E). No significant associations were identified between FOXN3 and other clinical parameters, including age, sex, hemoglobin or platelet count.

Association of FOXN3 expression levels with the survival of patients with AML. The association of FOXN3 expression levels with the treatment outcome of AML was investigated in a cohort of patients with the non-M3-subtype. A total of 45 patients were divided into a sub-group with decreased FOXN3 expression levels $(n=22)$ and a sub-group with increased FOXN3 expression levels $(n=23)$ according to the median level of FOXN3 expression (0.505; data not shown). The CR rate in the FOXN3 high-expression group $(n=19)$ was $88 \%$, whereas, the CR rate in the low-expression group $(n=17)$ was $77.2 \%$. No significant difference was observed in the $\mathrm{CR}$ rate between the two groups $(\mathrm{P}=0.608$; data not shown).

A 2-year follow-up was conducted on the 45 patients with the non-M3-subtype to study the effect of FOXN3 expression levels on the prognosis of patients with AML. The median survival time for patients was 13 months. There was no significant difference in the OS between patients with higher and lower FOXN3 expression ( $\mathrm{P}=0.2252$; Fig. 2A). However, a significantly decreased 2-year RFS was observed in patients with lower FOXN3 expression ( $\mathrm{P}=0.0177$; Fig. 2B). 

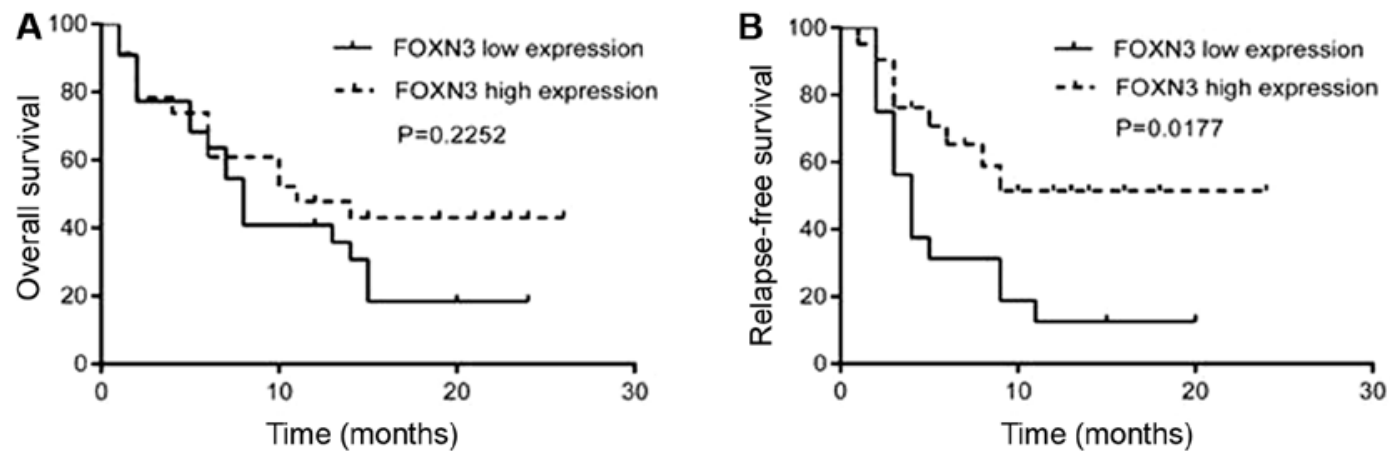

Figure 2. Association between FOXN3 expression levels and patient survival. (A) There was no significant difference in the 2-year overall survival rate of patients in the high and low FOXN3 expression groups. $P=0.2252$. (B) There was a significant difference in the 2 -year relapse-free survival rate between the patients in the high and low expression groups. $\mathrm{P}=0.0177$. FOXN3, forkhead box protein N3.

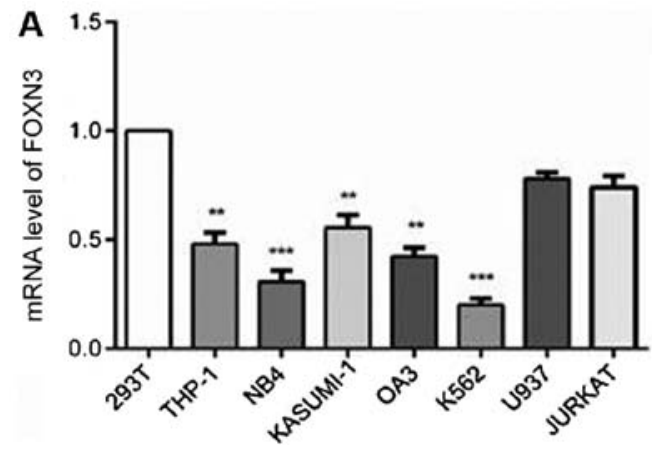

B

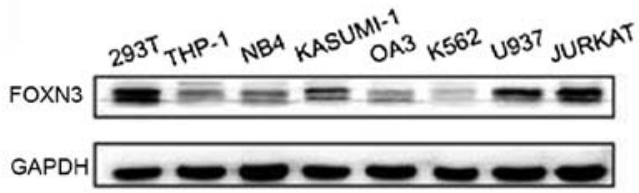

Figure 3. FOXN3 expression levels are decreased in AML cell lines. (A) FOXN3 expression levels were significantly decreased in five cell lines, OA3, NB4, K562, THP-1 and KASUMI-1 compared with the control 293T cells. ${ }^{* *} \mathrm{P}<0.01,{ }^{* * *} \mathrm{P}<0.001$ vs. 293T cells. (B) Western blotting of the FOXN3 expression levels in a panel of AML cell lines and the 293T control cells. FOXN3, forkhead box protein N3; AML, acute myeloid leukemia; OA3, OCI-AML3; THP-1, THP-AML.

AML cell lines exhibit decreased FOXN3 expression levels. The mRNA and protein expression levels of FOXN3 in leukemia cell lines, including THP-1, NB4, KASUMI-1, OA3, K562, U937 and JURKAT, and the control 293T cell line were detected using RT-qPCR and western blot analysis. Significantly decreased mRNA expression levels of FOXN3 were observed in all the AML cell lines tested, with the exception of the JURKAT and U937 cells, compared with the control 293T cells $(\mathrm{P}<0.01$; Fig. 3A). Correspondingly, decreased protein expression levels of FOXN3 were observed in the AML cells compared with the control 293T cells (Fig. 3B).

FOXN3 overexpression inhibits proliferation and promotes apoptosis. mRNA and protein expression levels of FOXN3 were increased in the FOXN3-transfected OA3 and THP-1 cells compared with the negative controls to study the role of FOXN3 in cell growth (Fig. 4A and B). Cell proliferation, cell cycle and apoptosis were measured by CCK- 8 and flow cytometry. FOXN3 overexpression significantly inhibited the proliferation of leukemia cells after 48 and $72 \mathrm{~h}$ compared with the control group $(\mathrm{P}<0.05$; Fig. $4 \mathrm{C}$ and $\mathrm{D})$. In addition, the total apoptosis (including early and late apoptosis) of leukemia cells increased following FOXN3 overexpression in OA3 cells (17.46 \pm 6.92 vs. $6.07 \pm 2.11 \%$; $\mathrm{P}<0.01$; Fig. $5 \mathrm{~A}$ and $\mathrm{B})$ and THP-1 cells $(12.99 \pm 1.19$ vs. $4.12 \pm 1.41 \%$; $\mathrm{P}<0.05$; Fig. $5 \mathrm{C}$ and $\mathrm{D}$ ). The proportion of S-phase cells increased significantly following FOXN3 overexpression in OA3 cells $(25.77 \pm 0.45$ vs. $16.76 \pm 0.37 \%$; $<<0.05$; Fig. $6 \mathrm{~A}-\mathrm{C})$ and THP-1 cells (29.33 \pm 0.47 vs. $13.42 \pm 0.47 \%$; $\mathrm{P}<0.05$; Fig. $6 \mathrm{D}-\mathrm{F})$.

\section{Discussion}

A large number of FOX transcription factors were demonstrated to serve important roles in tumorigenesis, including forkhead box protein $\mathrm{R} 1$, forkhead box protein $\mathrm{R} 2$ and forkhead box protein D1 (20-22). As a member of the FOXN subclass, FOXN3 is abnormally expressed in a variety of malignancies. FOXN3 may be a direct target of microRNA-574-5p, as it was downregulated by microRNA-574-5p to promote the progression of human lung cancer (23). A recent study on colon cancer demonstrated that the downregulation of FOXN3 activated the $\beta$-catenin/Wnt signaling pathway, thereby promoting colon cancer tumor cell growth, migration and invasion (13). In Hodgkin's lymphoma, FOXN3 may activate the Janus kinase-signal transducer and activator of transcription signaling pathways in transcriptional regulation (14). In addition, FOXN3 expression levels are decreased in a series of lymphomas, including primary exudative lymphoma, diffuse large B-cell lymphoma and hairy cell leukemia (14). The loss of FOXN3 led to an increase in the expression levels of the target gene serine/threonine-protein kinase Pim-2 (PIM2), thereby decreasing the effect of FOXN3 on tumor growth (24). However, the function of FOXN3 in AML has not yet been fully elucidated. 


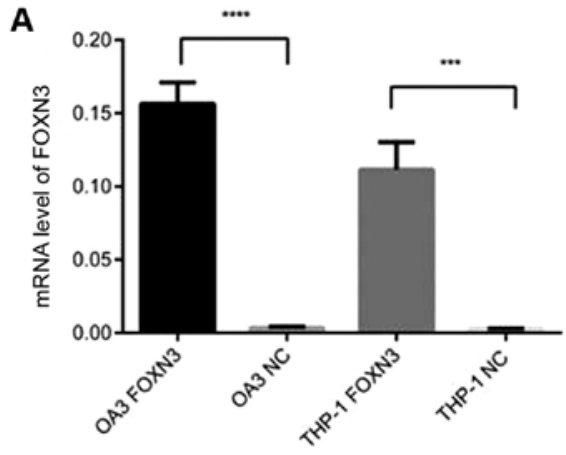

B
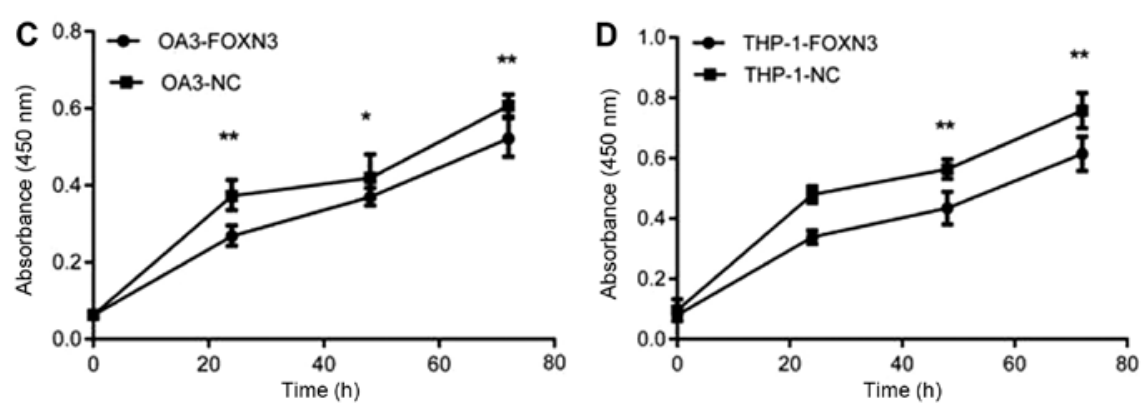

Figure 4. FOXN3 inhibits acute myeloid leukemia cell proliferation. Lentiviral transfection efficiency of FOXN3 as determined by (A) reverse transcription-quantitative polymerase chain reaction and (B) western blot analysis of OA3 and THP-1 cells. ${ }^{* * * *} \mathrm{P}<0.001,{ }^{* * * * *} \mathrm{P}<0.0001$. Proliferation analysis of $(\mathrm{C})$ OA3 and (D) THP-1 cells following transfection with FOXN3 lentivirus vector. ${ }^{*} \mathrm{P}<0.05,{ }^{* *} \mathrm{P}<0.01$ vs. respective NC. FOXN3, forkhead box protein $\mathrm{N} 3$; NC, negative control; OA3, OCI-AML3; THP-1, THP-AML.

A

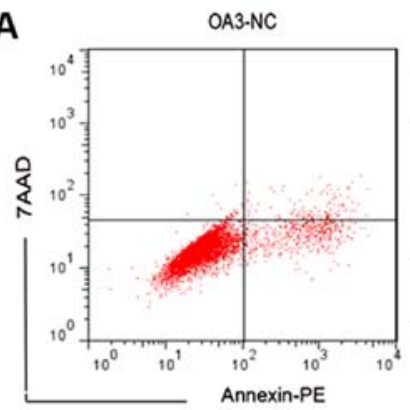

C

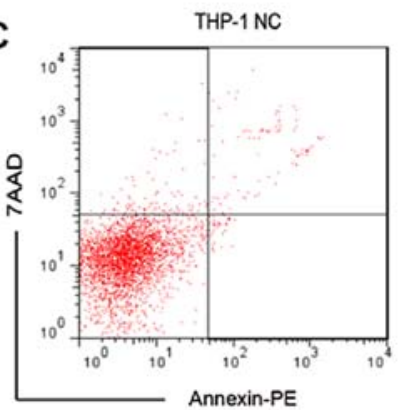

OA3-FOXN3

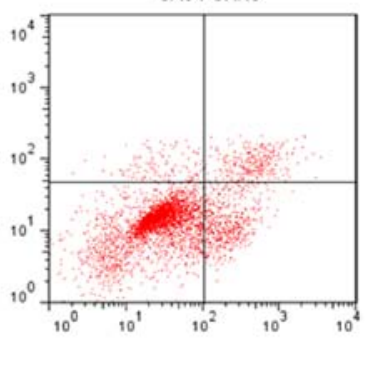

THP-1-FOXN3

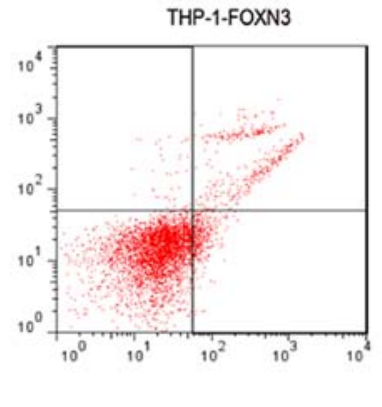

B
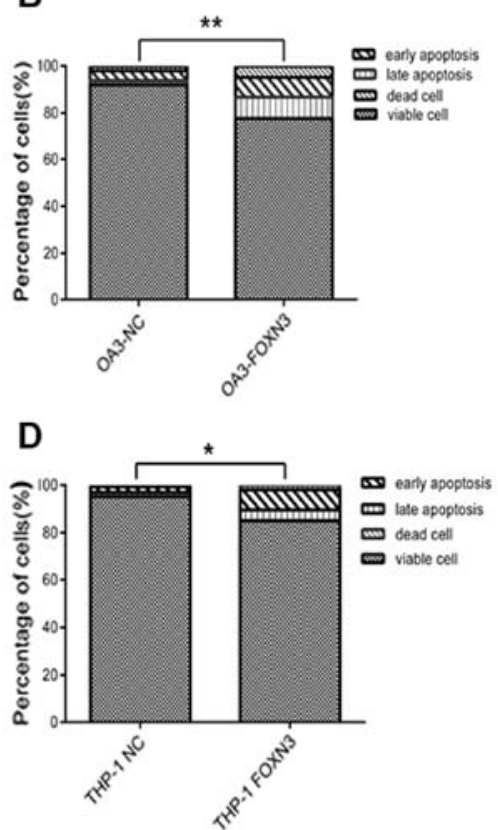

Figure 5. Apoptotic rates are significantly increased in cells transfected with FOXN3. (A) Scatterplots to determine the (B) apoptotic rate in OA3 cells overexpressing FOXN3. (C) Scatterplots to determine the (D) apoptotic rate in THP-1 cells overexpressing FOXN3. "P $<0.05$, "* P $<0.01$ for total apoptosis (early and late apoptosis). FOXN3, forkhead box protein N3; 7AAD, 7-amino-actinomycin D; PE, phycoerythrin; NC, negative control; OA3, OCI-AML3; THP-1, THP-AML.

In a previous study, high-resolution aCGH technology identified the presence of a FOXN3 gene deletion on chromosome 14 in two patients classified with M5-subtype AML (3), thus, the present study aimed to examine the potential role of FOXN3 in AML. M3 is a subtype of AML with aberrant $\mathrm{t}(15 ; 17)$ chromosome, serving as a treatment target. It is possible to cure the AML subtype using retinoic acid and arsenic containing compounds (25). At present, $95 \%$ of patients with M3 may achieve complete remission and long-term survival. The classification of AML as a M3 or non-M3-subtype deter- 

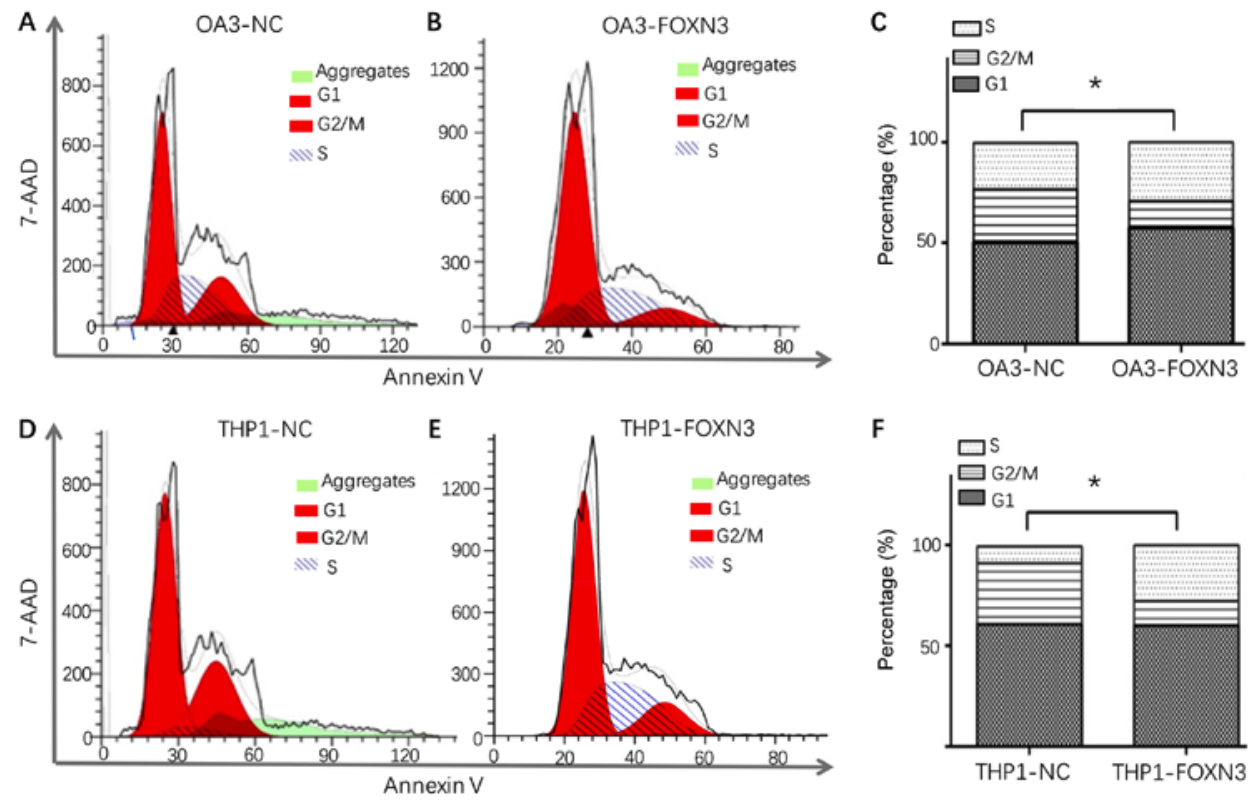

Figure 6. Cell cycle distribution following FOXN3 overexpression. Cell cycle distribution of the (A) OA3-NC cells and (B) OA3 cells with FOXN3 overexpression. (C) Apoptotic rates of OA3 cells were compared between the control and overexpression groups. Cell cycle distribution of (D) THP-1 NC cells and (E) THP-1 cells with FOXN3 overexpression. (F) Apoptotic rates of THP-1 cells were compared between the control and overexpression groups. "P $<0.05$ for proportion of S-phase cells. FOXN3, forkhead box protein N3; NC, negative control; OA3, OCI-AML3; THP-1, THP-AML; 7-AAD, 7-amino-actinomycin D.

mines the treatment regimen. FOXN3 expression levels were significantly decreased in patients with AML, particularly in patients classified with non-M3-subtype AML. Furthermore, the downregulation of FOXN3 was significantly correlated with the peripheral WBC count in patients, which is a high-risk prognostic marker of AML (26). This suggested that FOXN3 may be involved in the proliferation of leukemia cells. It was subsequently demonstrated that FOXN3 overexpression inhibited the malignant transformation of AML cells by decreasing cell proliferation, promoting apoptosis and enhancing cell cycle arrest. The prognostic significance of FOXN3 requires further investigation, as the downregulation of FOXN3 was associated with a decreased RFS, with no significant effect on OS. A possible explanation may be the regulatory effect of FOXN3 on cell proliferation, cell apoptosis and the cell cycle, suggesting the role of FOXN3 as a tumor suppressor in AML. Unlike RFS, the OS of AML is impacted by numerous factors, in addition to the relapse of leukemia. One possible explanation for the discrepancy was the association between lower FOXN3 expression levels and an increased WBC count, as the latter is considered a high-risk marker for relapse of leukemia (26).

However, the cellular signaling pathway regulated by FOXN3 in AML remains unknown. Scott and Plon (27) demonstrated that FOXN3 protein negatively regulated transcription through the $\mathrm{COOH}$ end of Ski-interacting protein (SKIP). SKIP inhibited tumor formation by competing with Ski oncogene to activate the transforming growth factor- $\beta$ (TGF- $\beta$ )/mothers against decapentaplegic homolog (Smad) signaling pathway (28). Previous studies suggested that the TGF- $\beta /$ Smad cell signaling pathway may regulate the proliferation and differentiation of hematopoietic stem cells, and is closely associated with the occurrence of leukemia (29-31). However, whether FOXN3 mediates hematopoiesis through the TGF- $\beta /$ Smad signaling pathway has not yet been determined $(32,33)$. It is hypothesized that the downregulation of FOXN3 may lead to the activation of the TGF- $\beta / \mathrm{Smad}$ signaling pathway and promote cell proliferation. This may result in hematopoietic stem cell proliferation and differentiation imbalances, which may induce leukemia. In addition, as a transcription inhibitor, FOXN3 binds directly to the promoter of PIM2 and decreases PIM2 expression (24), which is an important target of fms-like tyrosine kinase-3 (FLT3)-internal tandem duplication (ITD) induced transformation (34). PIM2 may complement the FLT3/wild-type (WT) signaling pathway and induce transformation similar to FLT3-ITD in myeloid cells (35). Furthermore, PIM2 and protein biosynthesis are principal targets of the antiproliferative effects of FOXN3 (24). Therefore, the downregulation of FOXN3 may increase PIM2 expression levels and promote leukemia transformation by activating FLT3/WT signaling.

The sample size was relatively small in the present study. Therefore, more patients are required for future studies to identify the effect of FOXN3 downregulation on the progression of AML. Furthermore, the use of in vivo experimental models is necessary to verify the tumor suppressive role of FOXN3 in AML. As a normal monocyte or granulocyte cell line was unavailable for the present study, the use of 293T cells as a control cell line was additionally a limitation.

In summary, FOXN3 was downregulated in AML. It may be a biomarker of high-risk AML, as the expression levels of FOXN3 were negatively correlated with peripheral WBC count and negatively associated with RFS in patients with AML. The contribution of FOXN3 to leukemogenesis may be through its regulatory effect on cell proliferation, apoptosis and the cell cycle. Whether FOXN3 affects any cellular signaling pathways, including the TGF- $\beta /$ Smad and FLT3/WT pathways, in AML requires further study. The results of the present study suggested that FOXN3 may be a novel therapeutic target as FOXN3 may be implicated in multiple signaling pathways associated with AML. 


\section{Acknowledgements}

Not applicable.

\section{Funding}

The present study was supported by the National Natural Science Foundation of China (grant no. 81600117).

\section{Availability of data and materials}

The datasets used and/or analyzed during the present study are available from the author on reasonable request.

\section{Authors' contributions}

HH collected the bone marrow samples of the patients, and analyzed and interpreted the data. JZ conducted the immunohistochemical study and follow-up of the patients. YQ conducted the cell experiments. YW performed the polymerase chain reaction assays. YZ was involved in the cell experiments. XY contributed to the data analysis. YL designed the research. $\mathrm{RZ}$ designed the study and was a major contributor in writing the manuscript. All authors read and approved the final manuscript.

\section{Ethics approval and consent to participate}

The present study was approved by The Ethics Committee of The First Affiliated Hospital of China Medical University (Shenyang, China). Prior to data collection, written informed consent for participation in the present study was obtained from each participant.

\section{Patient consent for publication}

Written informed consent for publishing the present study was obtained from each participant.

\section{Competing interests}

The authors declare that they have no competing interests.

\section{References}

1. Smith M, Barnett M, Bassan R, Gatta G, Tondini C and Kern W: Adult acute myeloid leukaemia. Crit Rev Oncol Hematol 50: 197-222, 2004

2. Siegel RL, Miller KD and Jemal A: Cancer Statistics, 2017. CA Cancer J Clin 67: 7-30, 2017.

3. Zhang R, Lee JY, Wang X, Xu W, Hu X, Lu X, Niu Y, Tang R, $\mathrm{Li} \mathrm{S}$ and $\mathrm{Li} \mathrm{Y}$ : Identification of novel genomic aberrations in AML-M5 in a level of array CGH. PLoS One 9: e87637, 2014.

4. Pati D, Keller C, Groudine M and Plon SE: Reconstitution of a MEC1-independent checkpoint in yeast by expression of a novel human fork head cDNA. Mol Cell Biol 17: 3037-3046, 1997.

5. Ostrow AZ, Kalhor R, Gan Y, Villwock SK, Linke C, Barberis M, Chen L and Aparicio OM: Conserved forkhead dimerization motif controls DNA replication timing and spatial organization of chromosomes in S. cerevisiae. Proc Natl Acad Sci USA 114: E2411-E2419, 2017.

6. Kaestner KH, Knochel W and Martinez DE: Unified nomenclature for the winged helix/forkhead transcription factors. Genes Dev 14: 142-146, 2000.
7. Schmidt J, Piekarski N and Olsson L: Cranial muscles in amphibians: Development, novelties and the role of cranial neural crest cells. J Anat 222: 134-146, 2013.

8. Schmidt J, Schuff M and Olsson L: A role for FoxN3 in the development of cranial cartilages and muscles in Xenopus laevis (Amphibia: Anura: Pipidae) with special emphasis on the novel rostral cartilages. J Anat 218: 226-242, 2011.

9. RobertT, Vanoli F,ChioloI,Shubassi G,Bernstein KA, Rothstein R, Botrugno OA, Parazzoli D, Oldani A, Minucci S, et al: HDACs link the DNA damage response, processing of double-strand breaks and autophagy. Nature 471: 74-79, 2011.

10. Cirillo LA and Zaret KS: Specific interactions of the wing domains of FOXA1 transcription factor with DNA. J Mol Biol 366: 720-724, 2007.

11. Busygina V, Kottemann MC, Scott KL, Plon SE and Bale AE: Multiple endocrine neoplasia type 1 interacts with forkhead transcription factor CHES1 in DNA damage response. Cancer Res 66: 8397-8403, 2006.

12. Sun J, Li H, Huo Q, Cui M, Ge C, Zhao F, Tian H, Chen T, Yao M and Li J: The transcription factor FOXN3 inhibits cell proliferation by downregulating E2F5 expression in hepatocellular carcinoma cells. Oncotarget 7: 43534-43545, 2016.

13. Dai Y, Wang M, Wu H, Xiao M, Liu H and Zhang D: Loss of FOXN3 in colon cancer activates beta-catenin/TCF signaling and promotes the growth and migration of cancer cells. Oncotarget 8: 9783-9793, 2017.

14. Nagel S, Meyer C, Kaufmann M, Drexler HG and MacLeod RA: Deregulated FOX genes in Hodgkin lymphoma. Genes Chromosomes Cancer 53: 917-933, 2014.

15. Mudduluru G, Abba M, Batliner J, Patil N, Scharp M, Lunavat TR, Leupold JH, Oleksiuk O, Juraeva D, Thiele W, et al: A Systematic Approach to Defining the microRNA Landscape in Metastasis. Cancer Res 75: 3010-3019, 2015.

16. Livak KJ and Schmittgen TD: Analysis of relative gene expression data using real-time quantitative PCR and the $2-\Delta \Delta C T$ method. Methods 25: 402-408, 2001.

17. Hsu SM, Raine L and Fanger H: Use of avidin-biotin-peroxidase complex (ABC) in immunoperoxidase techniques: A comparison between $\mathrm{ABC}$ and unlabeled antibody (PAP) procedures. J Histochem Cytochem 29: 577-580, 1981.

18. Zhu Y, Dai B, Zhang H, Shi G, Shen Y and Ye D: Long non-coding RNA LOC572558 inhibits bladder cancer cell proliferation and tumor growth by regulating the AKT-MDM2-p53 signaling axis. Cancer Lett 380: 369-374, 2016.

19. Zhu Z, Yang C, Wang J, Feng Q, Chen Q and Yang P: Altered chemokine receptor expression in the peripheral blood lymphocytes in polymyositis and dermatomyositis. Cytokine 99: 316-321, 2017.

20. Pan F, Li M and Chen W: FOXD1 predicts prognosis of colorectal cancer patients and promotes colorectal cancer progression via the ERK 1/2 pathway. Am J Transl Res 10: 1522-1530, 2018.

21. Katoh M, Igarashi M, Fukuda H, Nakagama $H$ and Katoh M: Cancer genetics and genomics of human FOX family genes. Cancer Lett 328: 198-206, 2013.

22. Wang X, He B, Gao Y and Li Y: FOXR2 contributes to cell proliferation and malignancy in human hepatocellular carcinoma. Tumour Biol 37: 10459-10467, 2016.

23. Li Q, Li X, GuoZ, Xu F, Xia J, Liu Zand Ren T: MicroRNA-574-5p was pivotal for TLR9 signaling enhanced tumor progression via down-regulating checkpoint suppressor 1 in human lung cancer. PLoS One 7: e48278, 2012.

24. Huot G, Vernier M, Bourdeau V, Doucet L, Saint-Germain E, Gaumont-Leclerc MF, Moro A and Ferbeyre G: CHES1/FOXN3 regulates cell proliferation by repressing PIM2 and protein biosynthesis. Mol Biol Cell 25: 554-565, 2014.

25. Lo-Coco F, Avvisati G, Vignetti M, Thiede C, Orlando SM, Iacobelli S, Ferrara F, Fazi P, Cicconi L, Di Bona E, et al; Gruppo Italiano Malattie Ematologiche dell'Adulto; German-Austrian Acute Myeloid Leukemia Study Group; Study Alliance Leukemia: Retinoic acid and arsenic trioxide for acute promyelocytic leukemia. N Engl J Med 369: 111-121, 2013.

26. Mamez AC, Raffoux E, Chevret S, Lemiale V, Boissel N, Canet E, Schlemmer B, Dombret H, Azoulay E and Lengliné E: Pre-treatment with oral hydroxyurea prior to intensive chemotherapy improves early survival of patients with high hyperleukocytosis in acute myeloid leukemia. Leuk Lymphoma 57: 2281-2288, 2016.

27. Scott KL and Plon SE: CHES1/FOXN3 interacts with Ski-interacting protein and acts as a transcriptional repressor. Gene 359: 119-126, 2005. 
28. Leong GM, Subramaniam N, Figueroa J, Flanagan JL, Hayman MJ, Eisman JA and Kouzmenko AP: Ski-interacting protein interacts with Smad proteins to augment transforming growth factor-beta-dependent transcription. J Biol Chem 276: 18243-18248, 2001.

29. Tabe Y, Shi YX, Zeng Z, Jin L, Shikami M, Hatanaka Y, Miida T, Hsu FJ, Andreeff M and Konopleva M: TGF- $\beta$-Neutralizing Antibody 1D11 Enhances Cytarabine-Induced Apoptosis in AML Cells in the Bone Marrow Microenvironment. PLoS One 8: e62785, 2013.

30. Su E, Han X and Jiang G: The transforming growth factor beta 1/SMAD signaling pathway involved in human chronic myeloid leukemia. Tumori 96: 659-666, 2010.

31. Kim SJ and Letterio J: Transforming growth factor-beta signaling in normal and malignant hematopoiesis. Leukemia 17: 1731-1737, 2003.
32. Zhou S, Fujimuro M, Hsieh JJ, Chen L, Miyamoto A, Weinmaster G and Hayward SD: SKIP, a CBF1-associated protein, interacts with the ankyrin repeat domain of NotchIC To facilitate NotchIC function. Mol Cell Biol 20: 2400-2410, 2000.

33. Vaidya A and Kale VP: TGF- $\beta$ signaling and its role in the regulation of hematopoietic stem cells. Syst Synth Biol 9: 1-10, 2015.

34. Hospital MA, Jacquel A, Mazed F, Saland E, Larrue C, Mondesir J, Birsen R, Green AS, Lambert M, Sujobert P, et al: RSK2 is a new Pim2 target with pro-survival functions in FLT3-ITD-positive acute myeloid leukemia. Leukemia 32: 597-605, 2018.

35. Agrawal S, Koschmieder S, Bäumer N, Reddy NG, Berdel WE, Müller-Tidow $C$ and Serve H: Pim 2 complements Flt3 wild-type receptor in hematopoietic progenitor cell transformation. Leukemia 22: 78-86, 2008. 